Revue d'histoire de l'Amérique française

REVUE D.HISTOIRE DE L'AMÉRIQUE FRANÇAISE

\title{
Entre l'art et la science : la littérature culinaire et la transformation des habitudes alimentaires au Québec
}

\section{Caroline Coulombe}

Volume 58, numéro 4, printemps 2005

La consommation

URI : https://id.erudit.org/iderudit/012211ar

DOI : https://doi.org/10.7202/012211ar

Aller au sommaire du numéro

\section{Éditeur(s)}

Institut d'histoire de l'Amérique française

\section{ISSN}

0035-2357 (imprimé)

1492-1383 (numérique)

Découvrir la revue

\section{Citer cet article}

Coulombe, C. (2005). Entre l'art et la science : la littérature culinaire et la transformation des habitudes alimentaires au Québec. Revue d'histoire de l'Amérique française, 58(4), 507-533. https://doi.org/10.7202/012211ar
Résumé de l'article

Cet article analyse l'évolution des usages alimentaires contemporains au Québec, à travers l'examen de la littérature culinaire domestique. Son objectif est de mettre en lumière les principaux changements qui s'opèrent dans l'univers culinaire contemporain, comme révélateurs de transformations plus profondes au sein de la société québécoise. Il démontre par le fait même la prégnance des nouvelles connaissances diététiques dans la transformation des habitudes de consommation ainsi que l'important processus de rationalisation de la cuisine, dont l'influence est à chercher du côté des principes d'organisation légués par l'ère industrielle. Nous constatons également l'émergence d'un discours gastronomique, où hospitalité, qualités artistiques et nouveautés alimentaires s'unissent pour offrir une vision renouvelée de la cuisine « moderne ". 


\section{Entre l'art et la science : la littérature culinaire et la transformation des habitudes alimentaires au Québec}

CAROLINE COULOMBE ${ }^{\mathrm{I}}$

Département des sciences humaines

Université du Québec à Trois-Rivières

RÉSUMÉ - Cet article analyse l'évolution des usages alimentaires contemporains au Québec, à travers l'examen de la littérature culinaire domestique. Son objectif est de mettre en lumière les principaux changements qui s'opèrent dans l'univers culinaire contemporain, comme révélateurs de transformations plus profondes au sein de la société québécoise. II démontre par le fait même la prégnance des nouvelles connaissances diététiques dans la transformation des habitudes de consommation ainsi que l'important processus de rationalisation de la cuisine, dont l'influence est à chercher du côté des principes d'organisation légués par l'ère industrielle. Nous constatons également l'émergence d'un discours gastronomique, où hospitalité, qualités artistiques et nouveautés alimentaires s'unissent pour offrir une vision renouvelée de la cuisine «moderne».

ABSTRACT - This article analyses the evolution of contemporary dietary practices in Quebec through a review of domestic culinary literature. Its purpose is to highlight the principal changes that have taken place in the contemporary culinary world, and to show how these changes are indicators of deeper transformations within Quebec society. As such, this article demonstrates the strong influence of new dietary knowledge on changes in consumption patterns, as well as the important process of the rationalization of cooking, which was influenced by the organizational principles inherited from industrialization. It also notes the emergence of a gastronomic discourse within which hospitality, artistic qualities and novelty foods come together to provide a renewed vision of «modern cuisine».

1. Nos remerciements vont à Paul-Louis Martin, Martine Tremblay et France Normand ainsi qu'aux évaluateurs de la Revue d'histoire de l'Amérique française pour leurs commentaires judicieux sur des versions préliminaires de cet article. 
Le champ de l'histoire de l'alimentation demeure peu couvert par les spécialistes des sciences humaines au Québec. Il constitue pourtant une porte d'entrée intéressante sur l'histoire du changement culturel. Dans le cadre de notre mémoire de maitrise ${ }^{2}$, nous avons eu l'occasion d'explorer quelques dimensions de la culture culinaire québécoise à travers une source principale très instructive: le livre de cuisine.

Depuis les années 1970 surtout, la question alimentaire a été examinée par à peu près tous les champs des sciences humaines et sociales. Cette recherche s'est inspirée, entre autres, des résultats de certains travaux incontournables dans le champ de l'anthropologie et de la sociologie culturelles $^{3}$. Bien qu'elles soient très éclairantes pour approfondir les concepts de goûts collectifs et de symbolisme alimentaire, les recherches issues de l'approche structuraliste se sont toutefois avérées peu enclines à considérer l'objet d'étude dans une perspective diachronique ${ }^{4}$. Les enquêtes historiques sur la consommation, dont plusieurs s'inscrivent dans la foulée de l'École des Annales, se sont quant à elles intéressées aux régimes alimentaires de diverses populations en négligeant la dimension culinaire ${ }^{5}$. Pour leur part, les travaux présentés dans l'ouvrage monumental dirigé par Jean-Louis Flandrin et Massimo Montanari rejoignent à plusieurs égards nos propres préoccupations, intégrant à la fois l'approche quantitative et comparative et l'étude des facteurs d'influence et des représentations collectives ${ }^{6}$.

2. Cet article est tiré de Caroline Coulombe, Un siècle de prescriptions culinaires : continuités et changements dans la cuisine au Québec, 1860-1960, mémoire de maittrise (études québécoises), Université du Québec à Trois-Rivières, 2002.

3. Les travaux du sociologue français Claude Fischler ont soulevé plusieurs questions intéressantes, notamment en ce qui a trait aux modalités du changement et à l'industrialisation de l'alimentation. Bien que sa démarche relève plutôt de la psycho-sociologie, sa présentation de la structure des repas et l'apparente dualité qu'il souligne entre gastronomie et santé ont contribué à enrichir considérablement notre propre réflexion. Claude Fischler, «Gastro-nomie et gastroanomie. Sagesse du corps et crise bio-culturelle de l'alimentation moderne», Communications, 31 (1979): 189-210. L'Homnivore. Le goût, la cuisine et le corps (Paris, Odile Jacob, 1990).

4. Entre autres, voir: Roland Barthes, «Pour une psycho-sociologie de l'alimentation contemporaine », dans J.-J. Hémardinquer, Pour une histoire de l'alimentation (Paris, A. Colin, 1970), 307-315; Pierre Bourdieu, La distinction. Critique sociale du jugement (Paris, Éd. de Minuit, 1979). Bourdieu s'est beaucoup intéressé à la différenciation sociale, alors que notre étude s'éloignait dès le départ d'une analyse basée sur le critère de classe sociale.

5. Entre autres Jean-Paul Aron, Essai sur la sensibilité alimentaire à Paris au XIx ${ }^{\mathrm{e}}$ siècle (Paris, A. Colin, coll. "Cahier des Annales», nº 25, 1967); J-J. Hémardinquer, op. cit., qui regroupe une série de travaux en histoire quantitative.

6. Jean-Louis Flandrin et Massimo Montanari, Histoire de l'alimentation (Paris, Fayard, 1996). Le second chapitre de notre mémoire inclut une analyse quantitative des ingrédients, par types, de près de 2500 recettes analysées. Cet exercice permet la comparaison dans le temps de leur usage. 
Les travaux récents dans le champ de l'histoire socioculturelle nous ont démontré l'influence des facteurs sociaux dans la transformation des habitudes alimentaires. Ce sont les études étasuniennes qui sont les plus fertiles sur la question du changement alimentaire pour le $\mathrm{xx}^{\mathrm{e}}$ siècle. Les travaux d'Harvey Levenstein ${ }^{7}$ sur les transformations du régime alimentaire américain depuis les années 1880 nous ont permis de situer l'expérience québécoise, notamment en regard des grandes tendances de consommation et de l'américanisation des habitudes alimentaires. D'autres travaux en histoire des femmes et plus récemment en histoire du genre ont considéré la question alimentaire comme partie prenante de l'univers domestique féminin et comme lieu d'expression de modèles sociaux et sexuels ${ }^{8}$. Une partie de la production québécoise s'est plus spécifiquement orientée vers l'étude de l'impact des aliments industriels sur le travail ménager des femmes ${ }^{9}$.

Pour notre part, nous nous sommes intéressée à la consommation alimentaire, plus particulièrement à l'étape de préparation de la nourriture: la cuisine. Le livre de cuisine s'est donc imposé comme un choix nécessaire pour une étude diachronique des pratiques culinaires et des goûts collectifs. Cet article propose une analyse de l'évolution des usages alimentaires contemporains au Québec, à travers l'examen de la littérature culinaire domestique publiée entre 1860 et 1960 . Son objectif est de mettre en lumière les principaux changements qui s'opèrent dans l'univers culinaire contemporain, comme révélateurs de transformations plus profondes au sein de la société québécoise. Il démontre par le fait même l'incidence des nouvelles connaissances diététiques dans la transformation des habitudes de consommation ainsi que l'émergence d'un discours gastronomique où hospitalité, qualités artistiques et nouveautés alimentaires s'unissent pour offrir une vision renouvelée de la cuisine «moderne».

7. Harvey Levenstein, Revolution at the Table. The Transformation of the American Diet (New York, Oxford University Press, 1988); Paradox of Plenty. A Social History of Eating in Modern America (New York, Oxford University Press, 1993).

8. Sherrie A. Inness, Kitchen Culture in America (Philadelphie, University of Pennsylvania Press, 2001); au Québec, Denise Lemieux et Lucie Mercier, Les femmes au tournant du siècle, 1880-1940 (Québec, Institut québécois de recherche sur la culture, 1989); Denyse Baillargeon, Ménagères au temps de la Crise (Montréal, Éditions du remue-ménage, 1991).

9. Suzanne Marchand, «L'impact des innovations technologiques sur la vie quotidienne des Québécoises au début du $\mathrm{Xx}^{\mathrm{e}}$ siècle (1910-1940)", Bulletin d'histoire de la culture matérielle, 28 (automne 1988): 1-14; Louise Fradet, Femmes, cuisine et consommation de masse au Québec, 1945-1960, mémoire de maîtrise (histoire) Université Laval, 1989; Tara Landry, Laveuse automatique et gâteau Duncan Hines: les impacts de la technologie domestique et des aliments à cuisson rapide sur la ménagère et sa représentation dans la publicité québécoise, mémoire de maitrise (histoire), Université du Québec à Montréal, 2000. 


\section{LA PÉRIODE}

La Chronologie du développement alimentaire au Québec ${ }^{10}$ met en lumière le chemin parcouru, depuis les débuts de la colonie jusqu'à aujourd'hui, tant au niveau de la variété et de la disponibilité des ressources naturelles, que de la culture matérielle, de l'avancement des connaissances en alimentation ou des technologies domestiques et industrielles. Si l'alimentation coloniale a fait l'objet de bon nombre d'études ${ }^{11}$, l'alimentation contemporaine a été beaucoup moins étudiée. La période 1860-1960 nous est d'emblée apparue intéressante, car elle témoigne du passage d'une société dite traditionnelle à une société de consommation et de production. En outre, l'analyse des prescriptions culinaires dans le long terme permet de saisir en partie l'évolution des goûts, l'introduction de nouveaux ingrédients et de nouvelles technologies, mais aussi les facteurs principaux qui ont induit les changements ou, au contraire, fait perdurer certains usages.

L'activité agricole est le pilier de notre système alimentaire. D’une agriculture de subsistance pratiquée par les premiers habitants de la colonie et plus tard par les colons des nouvelles régions, elle devint mar-

10. Claude Aubé, Chronologie du développement alimentaire au Québec (Saint-Jean-sur-Richelieu, Éd. du Monde alimentaire, 1996).

11. Au Québec, quelques études pionnières ont permis de connaître le régime alimentaire des habitants de la Nouvelle-France. La plupart ont été menées dans une optique plutôt descriptive, nourrissant un même postulat de l'uniformité des pratiques alimentaires. Charles-Marius Barbeau, "Ce qu'on mangeait autrefois», Cahiers de l'Académie canadienne-française, 9, (1944): 107-112; Robert-Lionel Séguin, «Le menu quotidien en Nouvelle-France», Liberté (janvier-février 1969): 65-90; La civilisation traditionnelle de l'habitant aux $\mathrm{XVII}^{\mathrm{e}}$ et $\mathrm{xVIII}{ }^{\mathrm{e}}$ siècles (Montréal, Fides, 1973), 701 p. ; Jean-Claude Dupont, Le pain d'habitant (Ottawa, Leméac, coll. "Traditions du geste et de la parole», 1974); aussi Le sucre du pays (Ottawa, Leméac, coll. «Traditions du geste et de la parole», 1975) et Le fromage de l'île d'Orléans (Ottawa, Leméac, coll. "Traditions du geste et de la parole», 1977). En fait, les études en histoire de l'alimentation au Québec ont peu tenu compte de la dimension de changement ou ont ignoré la période contemporaine, particulièrement le $\mathrm{xx}^{\mathrm{e}}$ siècle. L'étude de François Rousseau sur le régime des malades à l'Hôtel-Dieu de Québec, dont la problématique touche aussi bien à l'économie alimentaire qu'à l'univers des représentations, laisse de côté les facteurs de transformation de l'alimentation. Aussi, les sources premières de cette étude (des livres de comptes) renseignent davantage sur les aliments disponibles dans les réserves alimentaires que sur les plats cuisinés, puisque c'est du produit brut qu'elles rendent compte. François Rousseau, L'œuvre de chère en Nouvelle-France : le régime des malades à l'Hôtel-Dieu de Québec (Québec, Les Presses de l'Université Laval, 1983). L’ouvrage récent de Bernard Audet constitue une bonne synthèse des connaissances sur l'alimentation coloniale mais s'attarde surtout aux techniques de conservation et de transformation, rejoignant davantage le cadre de la culture matérielle. L'abondant recours aux sources secondaires ainsi que les nombreuses suppositions rendent à notre avis son "portrait des préférences alimentaires " plutôt statique, en plus de suggérer une certaine reproduction des pratiques métropolitaines françaises. Bernard Audet, Se nourrir au quotidien en Nouvelle-France (Sainte-Foy, Éditions GID, 2001). 
chande puis industrielle, repoussant toujours plus loin les limites de la productivité, devenant progressivement moins dépendante des aléas de la nature. La spécialisation des entreprises agricoles dès la fin du XIX ${ }^{\mathrm{e}}$ siècle a permis l'essor de l'industrie laitière, de l'élevage, de la culture maraîchère et fruitière et des grandes cultures. Si le nombre d'exploitations diminue tout au cours du $\mathrm{xx}^{\mathrm{e}}$ siècle, la production agricole québécoise offre un visage diversifié en 1960, bien que les importations d'aliments frais et transformés soient en hausse constante. Le secteur des aliments et boissons trône au premier rang de la valeur de la production industrielle québécoise durant toute la période, avec en tête les industries des abattoirs, de la salaison, du beurre et du fromage, la boulangerie ainsi que les brasseries et distilleries ${ }^{12}$. En outre, le développement des transports et des techniques de conservation a favorisé l'extension des marchés ${ }^{13}$, alors que le commerce a ressenti les effets tantôt favorables, tantôt défavorables de la conjoncture économique et politique. Parallèlement, des innovations dans la mise en marché ont rapproché les vendeurs des consommateurs qui ont accès, au tournant du siècle, à de nouvelles formes d'achat: catalogues, voyageurs de commerce, grands magasins. Vers le milieu du $\mathrm{xx}^{\mathrm{e}}$ siècle, les petits détaillants vont progressivement céder leur place aux succursales des grandes chaînes et aux supermarchés.

La période 1860-1960 est intéressante à d'autres égards. En plus de voir émerger une littérature culinaire propre au Québec, la deuxième moitié du XIX ${ }^{\mathrm{e}}$ siècle est propice à des percées importantes dans le domaine scientifique et particulièrement en diététique. Combiné à des efforts du milieu rural pour conserver les femmes dans leur rôle "traditionnel», ce courant favorise la création d'écoles d'enseignement ménager, dont la première a vu le jour à Roberval en 1882. Le mouvement d'enseignement ménager s'étendra assez rapidement au Québec, si bien qu'en 1930, on compte déjà 160 écoles ménagères dans la province.

$S$ 'ils offrent l'occasion aux femmes de sortir momentanément de leur rôle de maîtresse de maison, les conflits mondiaux contribuent aussi à modifier le rapport à la nourriture. En effet, les contraintes du rationnement sur certaines denrées combinées à la propagande efficace contre le gaspillage amènent les consommateurs à adapter leur régime alimen-

12. Paul-André Linteau, René Durocher, Jean-Claude Robert et François Ricard, Histoire du Québec contemporain, 2: Le Québec depuis 1930 (Montréal, Boréal Compact, 1989), 30, 61, 241-242.

13. Dès 1901 au Canada, des wagons réfrigérés transportent des aliments périssables. Carol Ferguson et Margaret Fraser, A Century of Canadian Home Cooking 1900 Through the '90s (Scarborough, Prentice Hall, 1992), 7. 
taire $^{14}$. Ces préoccupations, bien que temporaires, transparaissent dans les chroniques culinaires des journaux: les recettes économiques ${ }^{15}$ et les aliments de remplacement, comme le shortening ou les légumineuses sont mis de l'avant, quoique discrètement dans la littérature consultée.

Les générations de l'après-guerre ont assisté à une explosion de l'achat et de la consommation de produits et services. Le foisonnement des chroniques et des revues féminines, la diffusion des électroménagers dans les foyers et les changements apportés par la fin de la guerre (baby boom, développement des banlieues, hausse du niveau de vie) ont contribué à un renouveau culinaire marqué à cette époque. La dernière partie de cet article rend d'ailleurs compte de ce renouveau.

\section{LES SOURCES}

Selon l'historien Jean-Louis Flandrin, le livre de cuisine représente un lieu privilégié pour observer les goûts et les sensibilités qui deviennent en quelque sorte les normes dans la préparation de la nourriture. Un certain nombre d'auteurs ont investigué cette source pour différentes époques ${ }^{16}$. Marc Lafrance et Yvon Desloges ont, par exemple, regroupé des recettes tirées des répertoires culinaires français et anglais et consommées au Québec entre le $\mathrm{xvII}^{\mathrm{e}}$ et le $\mathrm{xIx}^{\mathrm{e}}$ siècles ${ }^{17}$. Cette enquête, qui a le mérite d'exploiter les livres de cuisine et de rendre compte du processus de canadianisation qui s'y manifeste, utilise surtout les manuels de la cuisine professionnelle, c'est-à-dire celle des grands chefs. Pour notre part, nous croyons que le recours à une littérature culinaire qui s'adresse avant tout

14. Sur le rationnement obligatoire aux États-Unis durant la Seconde Guerre mondiale, voir Amy Bentley, qui explore notamment le discours sur «the Wartime Homemaker». Amy Bentley, Eating for Victory. Food Rationing and the Politics of Domesticity (Urbana/Chicago, University of Illinois Press, 1998).

15. Une recette de pouding au pain à la crème au chocolat est présentée comme «recette économique de temps de guerre» dans une coupure de journal d'un cahier de recettes personnel (Montmagny, entre 1936 et 1945).

16. Du côté européen, les réceptaires du Moyen Âge et de l'époque moderne ont particulièrement retenu l'attention des chercheurs: Jean-Louis Flandrin, Chronique de Platine (Paris, Odile Jacob, 1992), 328 p. ; Carole Lambert, Du manuscrit à la table. Essais sur la cuisine au Moyen Âge (Montréal/Paris, Presses de l'Université de Montréal/Champion-Slatkine, 1992). Au Québec, le collectif Manger de France à Nouvelle-France regroupe des textes de chercheurs qui ont sondé la question alimentaire à partir du livre de cuisine, en particulier: Carole Lambert, «Survivance d'usages culinaires anciens dans la cuisine québécoise», dans Manger de France à Nouvelle-France (Québec, Société québécoise des ethnologues, 1990), 55-66 et Jocelyne Mathieu, «Emprunts anglo-saxons dans l'alimentation québécoise », dans Manger de France à Nouvelle-France, 89-93.

17. Marc Lafrance et Yvon Desloges, Goûter à l'histoire. Les origines de la gastronomie québécoise (Ottawa, Éd. de la Chenelière, Service canadien des parcs, 1989). 
aux femmes est essentiel pour connaitre les pratiques alimentaires de la société en général, et non pas uniquement celles d'une élite.

On pourrait d'emblée reprocher à ce type de document de ne présenter que la cuisine suggérée au lectorat féminin, et non celle effectivement pratiquée dans les foyers. Son contenu relevant du domaine du prescrit, il s'avère en effet difficile de savoir dans quelle mesure les recettes proposées étaient consommées par les ménagères et leurs familles. Or, les éditions successives de certains livres de recettes constituent des indices d'une adhésion populaire certaine. À titre d'exemple, les manuels publiés par la Congrégation Notre-Dame ont connu pendant longtemps une vaste diffusion, autant dans les écoles que dans les foyers québécois. D’ailleurs, la transmission des savoirs et des savoir-faire culinaires au Québec s'est surtout réalisée par l'intermédiaire des congrégations religieuses et de leurs écoles d'enseignement ménager. Une étude de l'évolution des pratiques alimentaires québécoises ne peut donc négliger, à notre avis, leur apport à notre culture culinaire. Ces dépositaires d'un savoir alimentaire ont laissé un riche héritage de connaissances, codifiées à l'intérieur de manuels publiés dès le milieu du $\mathrm{xIX}^{\mathrm{e}}$ siècle ${ }^{18}$. La série la plus populaire demeure sans contredit La cuisine raisonnée, dont la première édition remonte à 1919; des milliers de ménagères québécoises y ont puisé l'inspiration nécessaire pour étoffer leur répertoire culinaire personnel. Comptant près de vingt rééditions, ce livre témoigne très certainement de l'intérêt d'une large part du lectorat féminin pour les recettes et préceptes qui y sont présentés. C'est pourquoi il s'est avéré pertinent d'inclure dans notre corpus quelques éditions de cet ouvrage majeur, en l'occurrence la première édition de 1919 ainsi que celles de 1945 ( $5^{\mathrm{e}}$ édition) et 1954 ( $7^{\mathrm{e}}$ édition). Nos sources ont aussi compté sur quelques manuels d'économie domestique, sur des livres d'auteur et des productions gouvernementales.

Le développement de l'industrie alimentaire favorise l'émergence, vers 1920, d'un autre type de littérature culinaire, bon marché et abondant: les livres de compagnies alimentaires. L'objectif premier de ces fascicules étant évidemment la promotion de leurs produits, les industries investissent dans le développement de recettes qui les utilisent systématiquement

18. Le premier livre de recettes d'origine canadienne-française a été publié en 1840 sous le titre La cuisinière canadienne. Avant cette date, les traités culinaires qu'on retrouve dans la colonie proviennent essentiellement de France, d'Angleterre et des États-Unis. Pour notre étude, nous avons pu compter sur Directions diverses données par la Rév. Mère Caron pour aider ses soeurs à former de bonnes cuisinières (Montréal, Sœurs de la Providence, 1878). Selon Lafrance et Desloges (op. cit., 102), ce livre rend compte d'un "processus de canadianisation» de la cuisine assez manifeste. 
(farine, gélatine, cuisinière électrique pour n'en nommer que quelquesuns). On peut croire que plusieurs ménagères se sont procuré et ont essayé ces recettes facilement disponibles (souvent gratuites à l'achat du produit ou disponibles par commande postale). À cet égard, La cuisinière Five Roses, dont la première édition est sortie en $1915^{19}$, est un titre retrouvé dans plusieurs ménages. Les catégories précédentes couvrent selon nous les différents types de littérature culinaire disponibles durant cette période. Évidemment, d'autres titres peuvent paraître incontournables au lecteur, mais devant l'abondance des sources, un choix s'imposait.

Partant de l'évidence qu'une recette est d'abord préparée, mais éventuellement consommée, il importe également de prendre en considéra-

TABLEAU I

Liste des livres de cuisine consultés

\begin{tabular}{lcc}
\hline \multicolumn{1}{c}{ Titre } & Année(s) & $\begin{array}{c}\text { Type de } \\
\text { source }\end{array}$ \\
\hline Directions diverses données par la Révérende Mère Caron & 1878 & LI/MS \\
La bonne ménagère & 1896 & LI/MS \\
Hygiène de l'alimentation et propriétés chimiques des aliments & 1912 & LA \\
La cuisinière Five Roses (sélection) ${ }^{2}$ & 1915 & LC \\
Art culinaire: différentes manières d'utiliser les restes & 1916 & LI/MS \\
Manuel de cuisine raisonnée & 1919 & LI/MS \\
Consommons du lait & 1924 & PG \\
L'économie domestique à l'école primaire & 1925 & LI/MS \\
La cuisine à l'école complémentaire & 1939 & LI/MS \\
Menus balancés pour nos maisons d'éducation & 1944 & LI/MS \\
Le livre de cuisine de l'École de cuisine de Boston (sélection) & 1945 & LA \\
La cuisine raisonnée (sélection) & 1945 & LI/MS \\
Cuisine typiquement canadienne & $1945-1952 ?$ & LC \\
Recettes choisies Robin Hood & 1947 & LC \\
Cuisinez à l'électricité & $1948-1960 ?$ & LC \\
Exercices pratiques d'art culinaire: 8 $^{e}, 9^{e}, 10^{e}, 11^{e}$ années & 1948 & LI/MS \\
La cuisine raisonnée (sélection) & 1954 & LI/MS \\
La cuisinière Five Roses (sélection) & 1959 & LC \\
Art culinaire & 1960 & PG \\
\hline
\end{tabular}

1. Type de source: LI/MS: livre d'institution/manuel scolaire; LC: livre de compagnie alimentaire; PG: production gouvernementale; LA: livre d'auteur.

2. (Sélection): livres dont on a sélectionné les recettes.

19. Son prix de détail était de $0,30 \$$ en 1915. La cuisinière Five Roses (Montréal/Winnipeg, Lake of the Woods Milling co. 1td, 1915). 
tion les choix personnels et familiaux qui guident les ménagères dans la sélection des plats qu'elles cuisinent. C'est donc par souci de rendre notre corpus plus représentatif d'une réalité de consommation que nous avons demandé à cinq informatrices d'effectuer une sélection de recettes à l'intérieur de certains volumes préalablement identifiés ${ }^{20}$. Nous croyons que ce choix personnel, parmi des centaines de pages, des recettes les plus connues ou les plus appréciées dans leur jeunesse ou lorsqu'elles sont devenues mères de famille (elles sont nées entre 1916 et 1936), contribue à assurer la crédibilité de notre corpus. Dans le même esprit, sept cahiers de recettes manuscrits, récoltés dans des archives publiques ou personnelles, ont aussi été compilés.

Finalement, il ne faut pas oublier un autre vecteur important des discours sur l'alimentation et la cuisine : la presse. En effet, plus que les livres de recettes, les chroniques culinaires de journaux ont joué un rôle majeur dans la diffusion des tendances culinaires en vogue, à une époque où la presse écrite, particulièrement la presse populaire, connaît un essor impor$\operatorname{tant}^{21}$. Les pages féminines consacrent presque toujours un espace aux recettes, en plus de laisser la place à un discours culinaire souvent très éclairant sur les principes et les pratiques en vigueur à chaque époque ${ }^{22}$.

L'étude repose donc sur l'analyse d'une trentaine de titres et sur la compilation systématique de chaque ingrédient dans près de 2500 recettes tirées des manuels et des rubriques culinaires. Elle s'enrichit aussi d'une analyse des discours véhiculés dans ces sources.

Il est difficile d'établir de façon précise qui étaient les lectrices de nos sources $^{23}$. Le taux d'alphabétisation au Québec, qui connaît une croissance marquée, passant de $40 \%$ entre 1860 et 1869 à près de $80 \%$ entre 1890 et

20. Ces recettes «sélectionnées» représentent environ $10 \%$ de notre corpus total, puisées à même les manuels les plus volumineux.

21. Paul-André Linteau, René Durocher et Jean-Claude Robert, Histoire du Québec contemporain, 1:1867-1930 (Montréal, Boréal Express, 1979), 400.

22. Nous avons consulté La Gazette des campagnes, journal agricole publié à Sainte-Anne de La Pocatière de 1861 à 1895 et de 1941 à 1956, et Le Peuple, fondé à Montmagny en 1900. Ces deux journaux régionaux ont certes connu une diffusion limitée, mais ce choix visait surtout à recueillir des recettes accessibles à une majorité de ménages ruraux. Précisons que notre étude des prescriptions culinaires ne s'inscrit pas à proprement parler dans un cadre rural ou urbain, car plusieurs des titres sélectionnés ont connu une diffusion dans toute la province et même au Canada anglais. Il faut toutefois souligner que nos cinq informatrices habitent la Côte-du-Sud et que les cahiers de recettes manuscrits ont aussi été recueillis dans cette région.

23. Les sources retenues et l'approche choisie n'ont pas permis de dresser le profil social des lectrices. Une étude plus poussée nous aurait autorisée à analyser le genre de clientèle fréquentant les établissements ménagers et permis de connaitre en partie qui était le lectorat de cette littérature culinaire institutionnelle. 
$1899^{24}$, pour devenir universel après 1900 , et les coûts modestes de ce genre de littérature ${ }^{25}$ permettent vraisemblablement à un nombre croissant de lectrices d'avoir accès à la production sur l'art culinaire.

\section{LA SCIENCE AU SERVICE DE LA TABLE}

\section{Modèles diététiques}

Les découvertes scientifiques et leur diffusion par les spécialistes de l'alimentation ont largement contribué à faire évoluer les habitudes de consommation et ont permis l'émergence de nouveaux comportements alimentaires. Les sources ont révélé le rôle majeur que les hygiénistes, diététiciens et économistes domestiques ont joué dans la promotion d'une cuisine saine, basée sur les principes scientifiques en vigueur à chaque époque. Des livres comme ceux de l'École de cuisine de Boston ${ }^{26}$ ou Hygiène de l'alimentation et propriétés chimiques des aliments ${ }^{27}$ annoncent d'entrée de jeu un contenu qui s'appuie sur des principes diététiques reconnus. Au $\mathrm{xx}^{\mathrm{e}}$ siècle, les compagnies alimentaires recrutent les services d'économistes domestiques et d'autres spécialistes et élargissent leurs tests en cuisine $^{28}$. Même les manuels des congrégations religieuses, qui sont plutôt conservateurs dans leurs recettes, prennent le virage scientifique en mettant de plus en plus de l'avant les préceptes diététiques en vogue.

C'est dans la première décennie $d u \mathrm{xx}^{\mathrm{e}}$ siècle que la nutrition est reconnue comme discipline indépendante et scientifique ${ }^{29}$. Déjà au XIX ${ }^{\mathrm{e}}$, les progrès de la chimie alimentaire permettent d'identifier certaines substances nutritives: protéines, graisses, glucides, minéraux et eau sont considérés comme les cinq composantes essentielles des aliments. La décennie 1890 voit surgir un concept baptisé New Nutrition. Aux ÉtatsUnis, les réformateurs de la New England Kitchen «voulurent rationaliser les pratiques alimentaires de la classe ouvrière américaine, qu'ils jugeaient, à la lumière de la science nutritionnelle naissante, diététiquement et éco-

24. Michel Verrette, L'alphabétisation au Québec, 1660-1900, thèse de doctorat (histoire), Université Laval, 1989.

25. Entre $0,15 \$$ et $0,50 \$$ pour les manuels dont le prix nous était connu.

26. Porte-étendard des principes de la New Nutrition diffusés par la New England Kitchen, ce livre publié pour la première fois en 1896 et ses éditions subséquentes ont été l'un des titres les plus populaires aux États-Unis et probablement au Québec, puisque nous l'avons retrouvé dans plusieurs foyers. Fannie Merritt Farmer, Le livre de cuisine de l'École de cuisine de Boston (Toronto, $1945,7^{\mathrm{e}}$ éd.).

27. Amélie Desroches, Hygiène de l'alimentation et propriétés chimiques des aliments (Neuville, 1912).

28. C. Ferguson et M. Fraser, op. cit., 124.

29. Ibid., 8. 
nomiquement inadéquates ${ }^{30}$ ». L'entreprise échoua dans cette couche de la population, mais eut par contre des impacts assez profonds chez les classes moyennes. Ce courant sonna l'ère des calories: on pouvait désormais mesurer l'énergie fournie par chaque aliment.

La littérature culinaire québécoise rend compte de cette tendance et fournit une bonne idée de l'évolution du statut de plusieurs denrées, notamment le sucre. Aliment énergétique par excellence, il est considéré à la fin du $\mathrm{xIx}^{\mathrm{e}}$ siècle et au début du $\mathrm{xx}^{\mathrm{e}}$ siècle comme un véritable aliment. Son grand pouvoir calorifique sert d'incitatif à sa consommation et justifie son emploi. À preuve, une publicité de la compagnie Sun Maid, parue dans un journal en 1919 , soutient que ses raisins secs contiennent $75 \%$ de sucre et qu'ils procurent plus d'éléments nutritifs et d'énergie qu'une masse équivalente de bifteck ${ }^{31}$ ! Dans les décennies suivantes, le discours tend à se modérer: la plupart des manuels culinaires reconnaissent toujours le pouvoir énergétique du sucre, tout comme son utilité en cuisine, mais sensibilisent aussi la ménagère aux dangers d'en abuser ${ }^{32}$. Le sucre, et c'est là un des changements majeurs que nous avons pu observer, est progressivement évacué du modèle de consommation alimentaire équilibré proposé aux familles. D’ailleurs, ces discours trouveront plus tard leur concrétisation ultime dans un courant baptisé Negative Nutrition, qui émerge aux États-Unis dans les années $1960^{33}$. Paradoxalement, l'évolution des connaissances et l'apparition de discours visiblement plus "restrictifs » s'accompagnent d'une hausse du sucre, des desserts et des friandises dans les recettes que nous avons analysées. Le sucre passe ainsi de $42 \%$ à $52 \%$ du total des plats analysés entre 1860 et 1960. Le dessert, sucré il va sans dire, occupe une place prépondérante dans la cuisine québécoise. Si ces recettes représentent le quart du corpus de recettes analysées en 1860, elles atteignent le tiers d'entre elles en 1960, et cela même si le dessert n'est que le dernier service du repas. Pour leur part, les friandises comptent pour $1 \%$ des recettes avant $1890^{34}$ et pour plus de $4 \%$ après $1920^{35}$.

30. Claude Fischler, L'Homnivore, op. cit., 150.

31. Le Peuple, 21 mars 1919, 2.

32. D'ailleurs, cette substance a de tout temps endossé un statut ambigu: à la fois craint et recherché, dangereux et aphrodisiaque. Pour une histoire magistrale du sucre, voir Sidney Mintz, Sweetness and Power: The Place of Sugar in Modern History (New York, Viking, 1985).

33. Dans la foulée de ce courant, une série de combats s'entamera alors contre le sucre, le cholestérol, l'obésité, bref contre tous les excès de consommation jugés nuisibles à la santé publique.

34. Aucune recette entre 1890 et 1920.

35. Sur les friandises et leur place dans la culture culinaire américaine, voir Jane Dusselier, «Bonbons, Lemon Drops, and Oh Henry! Bars. Candy, Consumer Culture, and the Construction of Gender, 1895-1920», dans Sherrie A. Inness, op. cit., 13-49. 
De façon globale, la radicalisation du discours sur le sucre s'accompagne d'une hausse de son usage dans les recettes, qui est sans doute un bon indice d'une progression de sa consommation dans les foyers. Nous aborderons plus loin la dimension sociale associée à la consommation du sucre.

FIGURE I

Sucre, desserts et friandises dans les recettes

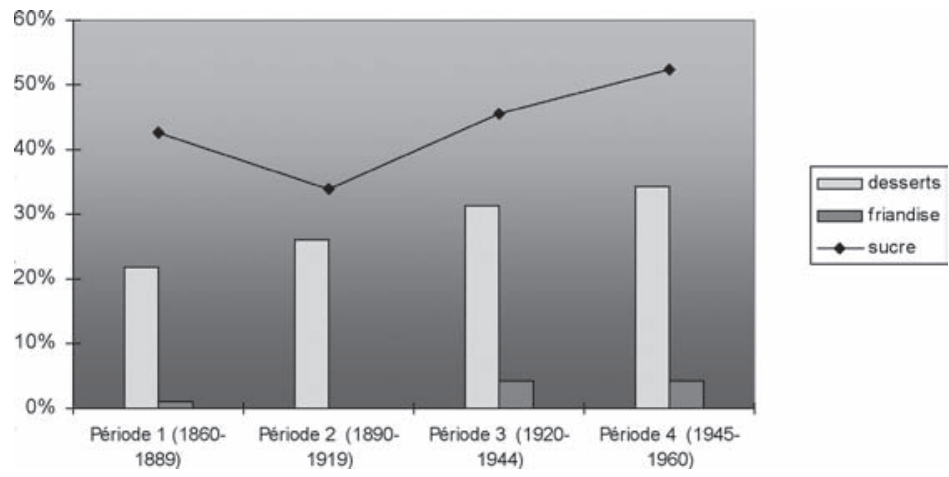

Durant la période, on accorde également beaucoup d'importance à la capacité nutritive et à la digestibilité des aliments. Tout cela se traduit, dans la littérature culinaire, par une attention nouvelle portée au calcul des éléments nutritifs nécessaires à une prise journalière équilibrée. Vers la fin du $\mathrm{xIX}^{\mathrm{e}}$ siècle, on sait que les corps gras fournissent les lipides nécessaires à la production d'énergie. On leur attribue alors une fonction essentielle dans le processus d'assimilation des aliments en facilitant leur digestibilité ${ }^{36}$. Dès avant le tournant $\mathrm{du} \mathrm{xx}^{\mathrm{e}}$ siècle, on remarque un souci de substituer aux matières grasses existantes - en particulier le saindoux - de nouveaux produits végétaux, réputés plus sains, appétissants et digestibles $^{37}$. Encore une fois, c'est la modération dans l'usage qui doit être la règle, car on connaît de mieux en mieux les effets de l'abus de gras sur la santé.

Par contre, dans les premières décennies $d u x^{e}$ siècle, la friture est considérée comme un mode de cuisson nutritif. Frire une pièce de viande ou un légume permet de conserver sa saveur et ses principes nutritifs, car l'albumine est coagulée à haute température, ce qui empêche les fluides

36. "Les assaisonnements de la cuisine et leur influence», La Gazette des campagnes, 8 février $1883,221$.

37. Publicité de la graisse végétale "cottolène», La Gazette des campagnes, 8 mars 1894, 416, 15 mars $1894,8$. 
intérieurs de s'échapper et les liquides extérieurs de pénétrer la pièce frite $^{38}$. L'aliment conserverait ainsi ses propriétés originelles, selon les croyances. Au fil des décennies toutefois, les prescriptions culinaires auront tendance à privilégier des types de cuisson au four ou sur la cuisinière plutôt que la cuisson en huile profonde.

L'usage des corps gras connaît une hausse encore plus marquée que le sucre dans les recettes: il passe de $48 \%$ en 1860 à $69 \%$ en 1960, encore que l'emploi de certains gras recule. La popularité des graisses animales diminue de moitié entre le $\mathrm{xIx}^{\mathrm{e}}$ et le $\mathrm{xx}^{\mathrm{e}}$ siècle ${ }^{39}$. Le lard, grâce surtout aux populaires grillades de lard salé, maintient sa popularité dans les plats principaux, mais suit la même courbe que les autres graisses animales, passant de $11 \%$ à $7 \%$ dans l'ensemble des plats. Encore une fois, les résultats livrent le constat d'une augmentation de l'usage des matières grasses, en même temps que s'accentue le discours sur les problèmes de santé reliés à leur consommation. Il faut toutefois souligner que l'évolution du choix des types de gras témoigne d'une préoccupation diététique certaine: les graisses animales sont plus souvent délaissées au profit de graisses végétales. L'embonpoint fait aussi plus fréquemment l'objet des chroniques culinaires des journaux au début de la décennie $1950^{40}$. Comme nous l'avons vu précédemment, les préceptes de la Negative Nutrition prennent forme à cette époque et n'iront qu'en s'accentuant.

L'observation de l'évolution du statut du sucre et du gras permet donc de constater qu'il existe un clivage entre le discours diététique, d'une part, et la consommation alimentaire, d'autre part, dont le portrait est en partie fourni par l'analyse de l'usage des ingrédients dans les manuels culinaires.

Dans la décennie 1920, un nouveau modèle alimentaire émerge avec la découverte des vitamines, qui révolutionnera les pratiques pour les décennies à venir ${ }^{41}$. Nommé Newer Nutrition, ce courant se manifeste de façon particulièrement précoce aux États-Unis mais ses influences se font sentir aussi assez rapidement dans la littérature culinaire québécoise. Autant les chroniques de journaux que les manuels d'art culinaire de l'époque récupèrent le nouveau modèle et en font un élément central dans le choix des

38. Congrégation Notre-Dame, La cuisine raisonnée (1919), 227.

39. De $31 \%$ entre 1860 et 1890 à $15 \%$ entre 1945 et 1960 .

40. "L'obésité constitue une grave menace pour la santé», La Gazette des Campagnes, 7 mai 1953, 5. Suzanne Marchand a par ailleurs constaté que le discours sur l'embonpoint est déjà présent dans la presse des années 1920-1930. Suzanne Marchand, Rouge à lèvres et pantalon (LaSalle/ Québec, Hurtubise HMH, 1997), 49.

41. Si la première vitamine est découverte dès 1912, c'est entre 1922 et 1926 que les recherches progressent véritablement. C. Ferguson et M. Fraser, op. cit., 54. 
aliments. Le concept d' "aliments protecteurs» est largement diffusé et fera école pendant longtemps. Dès lors, les aliments endossent moins un rôle curatif, comme c'était plus particulièrement le cas au XIX ${ }^{\mathrm{e}}$ siècle, que préventif $f^{42}$. Il est plus que jamais primordial de faire un choix éclairé parmi les groupes alimentaires suggérés, afin de fournir au corps tous les éléments nécessaires à sa défense ${ }^{43}$.

La littérature culinaire consultée a donc révélé la lente sensibilisation à un régime alimentaire privilégiant une consommation plus fréquente de végétaux et de fruits frais ou transformés, sans pour autant délaisser les menus à base de viande ${ }^{44}$. L'observation de l'utilisation des fruits et légumes dans les manuels culinaires montre comment le concept des vitamines a transformé les discours et les habitudes de consommation.

Si l'usage des végétaux demeure relativement stable dans les recettes entre 1860 et 1960, leur utilisation suscite un intérêt accru à en juger par les multiples accommodements dont ils font l'objet, à partir de 1920 surtout ${ }^{45}$. S'il était d'usage de cuire longuement les légumes avant de les consommer $^{46}$, le nouveau modèle imposé par les dernières connaissances diététiques suggère une diminution importante des temps de cuisson, jusqu'à cinq fois plus courts ${ }^{47}$, car on sait désormais que les vitamines sont détruites par l'action de la chaleur. Dans la même visée, la valorisation du "cru» marque les prescriptions culinaires du deuxième quart du $\mathrm{xx}^{\mathrm{e}}$ siècle. Les fruits, consommés cuits ou très mûrs avant 1920, ne sont pas réputés contenir beaucoup de nutriments. Les fruits crus en général sont même considérés peu digestibles. Nous n'avons d'ailleurs repéré aucune recette utilisant des fruits crus avant 1920. Le même phénomène est perceptible

42. Cette découverte permet d'aborder la maladie différemment. La théorie des germes, qui avait cours auparavant, voyait la maladie comme le résultat de l’ingestion d'agents étrangers dans l’organisme. Les traitements consistaient à bannir de l'alimentation certains aliments. La découverte des vitamines et minéraux impose une perception contraire, car la maladie résulte de l'absence de certains éléments. H. Levenstein, Revolution at the Table, op. cit., 148.

43. Élaborées en 1942 par le gouvernement canadien, les Règles alimentaires compilent les sept éléments essentiels d'une alimentation équilibrée: lait, fruits et légumes, produits céréaliers, viande, poisson, œufs, huile de foie de morue. Elles sont l'ancêtre du Guide alimentaire canadien.

44. "Au dire de certains hygiénistes, les légumes devraient former la base de notre alimentation». Congrégation Notre-Dame, L'économie domestique à l'école primaire (Montréal, 1925), $4^{\mathrm{e}}$ partie: Art culinaire, $2^{\mathrm{e}}$ leçon.

45. En salade, à la maître d'hôtel, au beurre, au jus, au lard, farcis, en purée, frits, en croquettes, beignets, croustades, ibid.

46. En raison notamment, d'une précaution diététique, qui était de débarrasser les légumes de toutes les substances jugées nuisibles à la digestion.

47. On recommande de cuire le chou, par exemple, de 8 à 20 minutes, alors qu'auparavant, on fixait son temps de cuisson à 2 heures en moyenne. 
pour la tomate, qui attend 1915 pour s'afficher «nature» dans une recette de club sandwich ${ }^{48}$. Les attitudes changent par la suite: salades, crudités et fruits "nature » sont régulièrement proposés aux ménagères, à côté des traditionnelles confitures et compotes. Cette constatation ne doit toutefois pas masquer une réalité sans doute fort répandue: la consommation des fruits et légumes crus en saison, phénomène que les livres de recettes ne peuvent malheureusement pas traduire. Les recettes témoignent par contre de l'utilisation croissante des agrumes entre 1920 et 1944. Une campagne intensive de promotion qui associe vitamine $\mathrm{C}$ et agrumes à cette époque n'y est sans doute pas étrangère. La consommation des agrumes et de leur jus au déjeuner semble bien installée dans les mœurs vers 1960 , si on en croit une brochure du Service de l'hygiène alimentaire canadien $^{49}$.

Enfin, même si les livres et les rubriques culinaires des journaux proposent des plats végétariens comme repas principaux, il reste difficile de mesurer cette tendance, puisque la nécessité d'observer le maigre du vendredi a depuis longtemps donné lieu à la création d'un répertoire de recettes sans viande. Le repas "végétarien " (c'est-à-dire "sans viande ») a d'abord été imposé par la pratique religieuse ou parfois par des considérations économiques. L’expression "végétarien» elle-même est relevée pour la première fois dans notre corpus dans les années 1940, puis à seulement quatre reprises par la suite, après 1950. L'existence d'un répertoire culinaire plus axé sur les végétaux semble témoigner d'un changement d'attitude envers le régime alimentaire à privilégier: au milieu du $\mathrm{xx}^{\mathrm{e}}$ siècle, c'est plus souvent par choix et par souci de santé qu'on confectionne salades et autres crudités. Le végétarisme sera d'ailleurs appelé à se développer en tant que véritable mode de vie alimentaire, dans la dernière moitié du $\mathrm{xx}^{\mathrm{e}}$ siècle.

L'hygiène fait également d'énormes progrès entre le milieu du $\mathrm{XIX}^{\mathrm{e}}$ et le milieu $\mathrm{du} \mathrm{xx}^{\mathrm{e}}$ siècle. Les découvertes antérieures de Pasteur sur la théorie des germes, tout comme les grandes épidémies du début du $\mathrm{xx}^{\mathrm{e}}$ siècle, ont certes contribué à légitimer les revendications du mouvement hygiéniste et à exacerber la phobie des germes qui se développe à cette époque. Ce phénomène mène à une véritable croisade sanitaire et touche autant l'aliment que l'environnement dans lequel il est préparé. Pour illustrer notre propos, nous observerons brièvement l'utilisation de cer-

48. La cuisinière Five Roses (1915), 27.

49. Six menus de déjeuner sur huit les proposent. Ministère de la Santé et du Bien-Être, Alimentation saine (Ottawa, Service de l'hygiène alimentaire, 1956). 
tains types de viande dans les recettes, les plus susceptibles selon nous de traduire les nouvelles sensibilités qui se développent dans ce domaine.

La présence des abats dans les livres de recettes connaît une diminution significative. Leur utilisation, qui s'élevait à $16 \%$ au XIX ${ }^{\mathrm{e}}$ siècle, chute à $7 \%$ dès le début $\mathrm{du} \mathrm{xx}^{\mathrm{e}}$ siècle. Le discours hygiénique, en plein essor, a dû contribuer à dévaloriser le statut de ces aliments, pourtant réputés très nutritifs ${ }^{50}:$ "les abats peuvent être le siège de nombreuses maladies parasitaires (ladrerie, trichinose, tuberculose) ${ }^{51}$ ». Quant au gibier, il chute de $10 \%$ à moins de $3 \%$ pour les mêmes périodes. La cuisine raisonnée recommande de consommer le gibier une fois son fumet développé, mais sans toutefois attendre le faisandage (qui est un début de putréfaction), comme c'était la coutume autrefois ${ }^{52}$. Visiblement, une méfiance se développe, dans la première moitié du $\mathrm{XX}^{\mathrm{e}}$ siècle, à l'égard des toxines potentiellement présentes dans ces viandes. De plus, les ménagères semblent perdre l'habitude d'apprêter les viandes sous leur forme entière, si l'on en juge par les illustrations détaillées des coupes et des étapes de dépeçage de différentes pièces de viandes qui s'introduisent dans les manuels. L'essor du commerce de détail et la possibilité de s'approvisionner chez le boucher ont dû contribuer à développer chez elles un certain dédain pour les travaux de boucherie maison.

Un manuel comme Hygiène de l'alimentation et propriétés chimiques des aliments $^{53}$ traduit d'ailleurs l'orientation que semble prendre la littérature culinaire du début du siècle. La "science ménagère » impose ses règles : la cuisine devient un laboratoire où la ménagère expérimentera diverses combinaisons alimentaires visant l'équilibre nutritionnel. Tous les domaines touchant à l'aliment semblent donc investis de cet idéal de santé, d'hygiène et de propreté. La "pureté» des aliments issus de l'industrie, produits dans des environnements hautement contrôlés et supposément exempts de germes, est un autre outil promotionnel utilisé par les compagnies alimentaires, et un fort incitatif à modifier les pratiques acquises.

En somme, pendant la période à l'étude, la santé devient un leitmotiv dans le discours culinaire, à un point tel que les entreprises alimentaires n'hésitent pas à y avoir recours pour promouvoir leurs produits. L'argu-

50. Source de fer et vitamines.

51. Congrégation Notre-Dame, La cuisine raisonnée (1954), chapitre 10: Étude des substances alimentaires.

52. Congrégation Notre-Dame, La cuisine raisonnée (1919), chapitre 22: Le gibier.

53. Amélie Desroches, op. cit. 
ment sert même parfois de justification à la consommation de substances à forte teneur en sucre ou peu nutritives: l'ajout de sucre, de friandises ou de colorant aux fruits, céréales et produits laitiers est régulièrement présenté, dans la presse, comme un moyen de faire consommer aux enfants les aliments sains. Certaines compagnies poussent l'audace jusqu'à employer des tactiques plus ou moins trompeuses, laissant croire aux qualités nutritives de leurs produits: Pepsi-Cola, en l'occurrence, affirme en 1943 qu'une bouteille de sa boisson gazeuse contient 173 calories, "presqu'autant $[$ sic $]$ qu'un bon morceau de viande ${ }^{54}$ ".

\section{L'ère de la rationalisation : produits industriels et cuisine standardisée}

Les cent ans qui couvrent la période 1860-1960 correspondent à une longue phase d'industrialisation et de mécanisation du travail. Durant cette période et sous l'impulsion de découvertes technologiques majeures, l'industrie alimentaire fait des progrès importants. Mais l'ère industrielle fait plus que développer le secteur de l'aliment «usiné». Elle lègue aussi des principes d'organisation appelés à influencer tous les secteurs de la vie sociale et économique, même l'univers domestique. Aliments industriels et cuisine standardisée témoignent tout particulièrement du processus de rationalisation qui s'opère dans la cuisine domestique contemporaine.

Les aliments industriels font leur apparition au sein d'une économie de marché en pleine expansion. Appelés produits à préparation rapide dans le jargon commercial, il s'agit de denrées semi-préparées, prêtes à utiliser et issues d'innovations techniques dans plusieurs secteurs de l'industrie alimentaire, notamment la conserve, popularisée ici avant la fin du XIX ${ }^{\mathrm{e}}$ siècle, l'emballage et la congélation. Comme l'a relevé Claude Fischler, l'industrialisation de l'alimentation s'est faite assez précocement aux ÉtatsUnis : des géants comme Coca-Cola, Nabisco, Heinz, Kellogg, Campbell sont déjà présents sur le marché à la fin du XIX ${ }^{\mathrm{e}}$ siècle ${ }^{55}$. Mais ces produits prendront quelques décennies à rejoindre une majorité de ménages au Québec. Ces nouveautés alimentaires ont pu se diffuser, entre autres, par le biais des livrets de recettes publiés par les compagnies alimentaires et surtout par les publicités et les chroniques culinaires de la presse écrite.

Une analyse des valeurs véhiculées par les publicités et les auteurs des chroniques culinaires, dans les années 1940-1950, met en lumière la primauté de la commodité et de l'efficacité comme incitatifs à la préparation

54. «Encore les liqueurs douces», La Gazette des campagnes, 25 mars 1943, 248-249.

55. Claude Fischler, «La "macdonaldisation" des mœurs» dans J.-L. Flandrin et M. Montanari, op. cit., 863 . 
et à la consommation des aliments industriels. À cet égard, le temps devient une denrée précieuse, signe de la transformation des modes de vie. L'argument de la diversité dans les menus est aussi utilisé en faveur des produits préparés. Se déclinant sous de multiples variantes et saveurs, les jus, soupes, crèmes, mélanges à desserts, gélatines, sauces et autres préparations permettent à la ménagère de gagner du temps tout en jouant la carte de la nouveauté.

La soupe en boîte est l'une de ces révolutions culinaires très estimées par les chroniqueures féminines du milieu du $\mathrm{xx}^{\mathrm{e}}$ siècle $^{56}$. Selon nous, cet exemple résume, à lui seul, l'essence même du discours sur l'alimentation «moderne». Relevées pour la première fois dans notre corpus en 1910, les soupes en boîte sont sensées représenter l'«idéal» de la cuisinière moderne. Cuisinées dans des environnements contrôlés avec des ingrédients sélectionnés, elles sont nourrissantes, rapides à préparer et s'accordent avec les habitudes de consommation où les soupes ouvrent habituellement les repas $^{57}$. La panoplie des saveurs disponibles croît à mesure que le marché se développe: crème de poulet et pois verts, crème de champignons et soupe à l'oignon, légumes à la végétarienne et bouillon ${ }^{58}$ donnent un aperçu des combinaisons suggérées dans les rubriques culinaires des journaux.

En plus de valoriser la diversité, le discours culinaire met l'accent sur l'aspect rationnel: les principes d'efficacité, de précision et de planification, associés au développement du travail à la chaîne et à la spécialisation des tâches, se sont transposés dans l'aire domestique dans la première moitié $\mathrm{du} \mathrm{xx}^{\mathrm{e}}$ siècle. La littérature culinaire a constitué un vecteur par lequel les nouvelles théories de l'organisation domestique se sont diffusées, et un témoin par excellence de l'impact de l'industrialisation sur la façon de cuisiner.

Une brève analyse de la morphologie des livres de cuisine met au jour une transformation plutôt subtile, autour de 1915-1920, de l'organisation des manuels culinaires. Alors que la description des ingrédients et des étapes de préparation demeure la plupart du temps très sommaire au XIX ${ }^{\mathrm{e}}$

56. Pour un aperçu du discours véhiculé par un géant de la conserve aux États-Unis, voir Katherine Parkin, "Campbell’s Soup and the Long Shelf Life of Traditional Gender Roles », dans Sherrie A. Inness, op. cit., 51-67.

57. La préparation d'une soupe maison nécessite beaucoup de temps, surtout dans les premières décennies de la période, alors que quatre recettes de soupes sur dix emploient la viande comme base de bouillon. Au milieu du xx ${ }^{\mathrm{e}}$ siècle, seulement une sur dix perpétue cet usage. Les potages à base de crème, de bouillon préparé et de tomate remplacent l'ancienne méthode.

58. «Emballez des lunchs qui plaisent à votre enfant», La Gazette des campagnes, $1^{\mathrm{er}}$ septembre 1955,4 . 
siècle, les livres du $\mathrm{xx}^{\mathrm{e}}$ siècle s'enrichissent progressivement de nouvelles indications: une liste des ingrédients dans leur ordre d'utilisation et des mesures de plus en plus précises remplacent le caractère plutôt approximatif de certains livres antérieurs et semblent devenir la norme dans la littérature culinaire après 1920.

En outre, le nombre moyen d'étapes requises dans la préparation des plats tend à s'allonger entre le milieu du $\mathrm{xIx}^{\mathrm{e}}$ et le milieu du $\mathrm{xx}^{\mathrm{e}}$ siècle. Les recettes courtes (cinq étapes et moins) se retrouvent légèrement majoritaires entre 1860 et 1890, alors que $60 \%$ des recettes longues (treize étapes et plus) sont relevées après 1945 . Cette augmentation des étapes de préparation se produit au moment même où triomphent les principes de rationalisation des usages et d'efficacité.

Deux hypothèses peuvent expliquer cette situation en apparence paradoxale. D’un côté, nous pouvons croire qu'il existe effectivement une tendance à la complexification de la cuisine : la confection de la nourriture demande plus de temps parce que les préparations sont davantage élaborées et les ingrédients plus nombreux. Pourtant, la commercialisation des produits préparés devait simplifier les opérations culinaires.

D’un autre côté, cette tendance témoigne d'une plus grande précision dans la rédaction des étapes de confection et va dans le sens d'une rationalisation accrue. Ce phénomène illustre probablement une perte de connaissances générales de la part des lectrices, à qui il faut désormais expliquer chaque geste ${ }^{59}$. Le resserrement des critères de précision, issu d'une approche de plus en plus scientifique de la cuisine, a probablement poussé les auteurs des manuels à s'assurer que les lectrices exécutent adéquatement et de façon précise chacune des étapes requises. Dans le même sens, la simplicité désarmante des recettes présentées dans les cahiers plus anciens semble confirmer que les ménagères d’antan savaient comment préparer les plats. Pouvons-nous parler d'une perte de savoirfaire entre le milieu du $\mathrm{xIx}^{\mathrm{e}}$ et le milieu du $\mathrm{xx}^{\mathrm{e}}$ siècle? Il semblerait que «les femmes non préparées aux tâches domestiques [soient] de plus en plus nombreuses en ce début de $\left[\mathrm{xx}^{\mathrm{e}}\right]$ siècle $^{60}$ ».

59. Tout porte à croire que les ménagères d’antan connaissaient déjà les étapes nécessaires. Ainsi, "faire une bonne pâte feuilletée» est une indication suffisante dans la Mère Caron, alors qu'au $\mathrm{XX}^{\mathrm{e}}$ siècle, toutes les étapes sont énumérées : "tamiser, incorporer, abaisser, etc. ».

60. Denise Lemieux et Lucie Mercier, op. cit., 306. Un journal agricole justifie le besoin d'un enseignement ménager dès 1878 : Il manque aux filles des champs la manière d’apprêter les mets. L'éducation des filles des cultivateurs mériterait de s'orienter davantage vers l'art culinaire plutôt que d'enseigner des futilités. Il reviendrait également aux mères de se faire un devoir d'enseigner à leurs filles les rudiments de la cuisine", La Gazette des campagnes, 7 mars 1878, 77-78. 


\section{L'ère de la diversité}

L'essor des technologies et des échanges commerciaux a favorisé l'avènement de la société de consommation et son corollaire, la diversification des produits du marché. Le phénomène est observable pour les produits issus de l'industrie, comme nous l'avons vu avec l'exemple des soupes en boîtes, mais il touche également les aliments bruts utilisés dans les recettes, en particulier les fruits et les légumes. La figure 2 rend compte de la variété des fruits et légumes utilisés dans les recettes entre 1860 et 1960. Dix-neuf sortes de légumes sont dénombrées avant 1920. Ce nombre s'élève à trente entre 1945 et 1960. Les variétés de fruits disponibles entre 1860 et 1960 croissent dans les mêmes proportions. Une diversité plus grande de végétaux sur les étals reflète les progrès des transports et de la conservation, mais signifie surtout, pour les consommateurs, une possibilité de choix accrue. Du côté des fruits, cette disponibilité nouvelle se traduit notamment par la croissance phénoménale des agrumes et autres espèces exotiques dans les recettes des manuels postérieurs à $1920^{61}$.

Cette tendance à la diversité rend compte d'un autre phénomène, à première vue paradoxal: l'uniformisation des usages. À l'instar de certains historiens de l'alimentation ${ }^{62}$, nous avons vu s'affirmer cette double ten-

\section{FIGURE 2}

Nombre d'espèces de fruits et de légumes

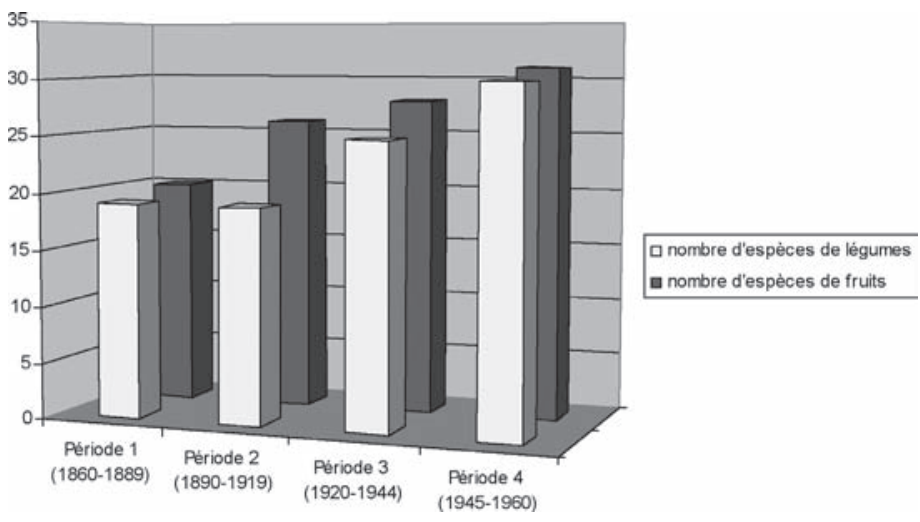

61. Un article du Peuple, en 1919, nous renseigne sur la disponibilité de certains fruits exotiques sur le marché de Québec: citron, orange, banane, pamplemousse, ananas, coco. «Le commerce à Québec », Le Peuple, 4 juillet 1919.

62. Harvey Levenstein parle d'une nationalisation des habitudes alimentaires américaines. Revolution at the table, op. cit., 170. Voir aussi Stephen Mennell, Français et Anglais à table du Moyen Âge à nos jours (Paris, Flammarion, 1987), chapitre 12. 
dance: à mesure que les réseaux de distribution s'étendent, que la production alimentaire se rationalise, que les aliments se diversifient et deviennent disponibles sur une plus longue période, la culture culinaire québécoise offre le portrait d'une consommation alimentaire moins contrastée et il devient possible de "consommer toute l'année un certain nombre de mets qu'on préfère ${ }^{63} »$. Cette tendance est d'autant plus forte que l'industrialisation de l'alimentation tend à produire des aliments de plus en plus standardisés dont l'aspect, la texture et le goût se caractérisent par une certaine uniformité. L'expérience québécoise s'inscrit donc dans une réalité occidentale largement influencée par ces phénomènes. Une étude de la consommation alimentaire régionale ou locale révélerait sans doute des particularités intéressantes.

\section{DES MODÈLES SOCIAUX ET CULTURELS EN MUTATION ?}

Au-delà des transformations technologiques et des nouvelles valeurs qu'elles mettent de l'avant, la cuisine québécoise subit l'influence de changements d'ordre culturel et social, particulièrement pour le deuxième quart du $\mathrm{xx}^{\mathrm{e}}$ siècle. Les livres de cuisine et les chroniques des journaux insistent sur le rôle traditionnel des femmes en tant que responsables des repas familiaux. Ils révèlent néanmoins à ce sujet un glissement d'idéal entre 1860 et 1960 : le savoir-faire et l'expérience cèdent le pas au paraître, à l'originalité et à la nouveauté.

\section{«Une bonne hôtesse tu seras»}

Les manuels de cuisine domestique compilent les connaissances et les techniques alimentaires, mais ils contribuent aussi à la diffusion du "modèle» de la parfaite cuisinière et de la femme idéale. En raison du public à qui ils s'adressent et parce qu'ils touchent à l'une de ses principales activités, leurs discours renferment les préceptes énoncés dans d'autres formes d'écrits spécifiquement destinés aux femmes: manuels d'enseignement ménager, revues féminines, publicités ${ }^{64}$.

Les discours culinaires contemporains célèbrent de plus en plus les qualités d'hospitalité de la maîtresse de maison. Car bien que la consommation de nourriture soit un acte d'abord destiné à satisfaire des besoins

63. Stephen Mennell, op. cit., 462.

64. À cet égard, l'histoire du mouvement d'enseignement ménager fournit un bon exemple de la pérennité d'un modèle féminin traditionnel. Nicole Thivierge, Histoire de l'enseignement ménager-familial au Québec 1882-1970 (Québec, Institut québécois de recherche sur la culture, 1982). Plusieurs travaux se sont aussi intéressés à l'univers domestique et aux discours sur les femmes durant la guerre. Voir notamment Amy Bentley, op. cit. 
physiologiques, dans nombre de cultures, les repas sont aussi un acte de sociabilité. Les chroniques féminines du milieu du $\mathrm{xx}^{\mathrm{e}}$ siècle intègrent particulièrement les lectrices dans l'univers moderne de la consommation: d'appareils ménagers, de denrées industrielles, mais surtout de relations interpersonnelles, valorisant à profusion les occasions de rencontre autour de la nourriture ${ }^{65}$. La bonne femme de maison acceptera de recevoir chez elle les copains de ses enfants après l'école, les collègues de son mari après le travail, la famille ou les amis pour les anniversaires ou les réunions dominicales. Nous percevons aussi, au cours de la période étudiée, une mutation du modèle de la "parfaite cuisinière» en celui de la "parfaite hôtesse ", les qualités sociales se substituant aux qualités essentiellement techniques. L'accueil des invités devient alors tout aussi important que la confection même de la nourriture. On encourage la femme à cuisiner pour plusieurs convives, non plus pour nourrir une famille nombreuse mais pour impressionner les invités. Derrière ces incitations à l'hospitalité se cachent autant d'occasions d'exposer certains signes de réussite: menus "de réception", barbecues et garden parties sont autant de formules qui assurent le succès des repas ${ }^{66}$.

Dans l'après-guerre, à la faveur du baby-boom, des transformations de la vie matérielle et du développement de la psychologie infantile, les besoins de l'enfant prennent une place déterminante ${ }^{67}$. Nous avons constaté que les discours des rubriques féminines sont imprégnés de ces nouvelles conceptions, qui véhiculent l'idéal de la ménagère accueillante et réconfortante. Les réceptions d'enfants, les réunions d'adolescents après l'école deviennent autant d'occasions pour la mère de servir de petites "douceurs»: beignes, laits frappés, friandises. On sent aussi une volonté de faire plaisir aux enfants en leur servant une nourriture attrayante, colorée et souvent sucrée. L’engouement croissant pour les aliments

65. Et non pas nécessairement autour de la table, qui n'est visiblement plus le seul lieu de prise alimentaire : la collation, qui fait l'objet dans les années 1950 d'un intérêt croissant, est particulièrement indiquée pour les enfants, qu'on autorise même à grignoter devant le téléviseur. Cet éclatement de l'espace de consommation est aussi à mettre en relation avec la popularité croissante du sandwich, qui se consomme n'importe où, sans assiette ni ustensiles.

66. Pour la ménagère, opter pour ces formes de repas est un moyen de montrer qu'elle adopte les dernières tendances. "Dans nombre de familles qui possèdent un jardin, les rôtisseries extérieures ont fait leur apparition selon la mode du grill qui sévit aux États-Unis depuis quelques années». «À table», Gazette des campagnes, 10 août 1956, 4. L'importance accordée à des pratiques de consommation «à la mode» est traitée un peu plus loin.

67. D’autres auteurs l'ont souligné: la fin des années 1940 et les années 1950 ont vu l'émergence d'une nouvelle forme de culture de consommation et d'une culture centrée sur les enfants, mais aussi sur la nourriture. Erika Endrijonas, «Processed Foods from Scratch. Cooking for a Family in the 1950's", dans Sherrie A. Inness, op. cit., 157. 
sucrés résulte sans doute en partie de ces préoccupations nouvelles pour les jeunes, dans la société de l'époque.

\section{Esthétique, gastronomie et aliments «à la mode»}

En affirmant l'importance de la commensalité, le discours culinaire rehausse du même coup l'importance des qualités artistiques en cuisine. On remarque, en effet, au cours des années 1950, une recherche plus poussée de l'esthétique culinaire et un intérêt croissant pour la présentation des mets, le plaisir visuel devenant presque plus important que le goût ${ }^{68}$. L'attrait pour le raffinement s'exprime tout d'abord à travers une présentation plus sophistiquée. C'est l'époque des gâteaux à étages multiples, des salades colorées, des galantines savamment décorées, des tomates taillées en forme de fleurs, etc. Cette tendance commande souvent l'usage de produits frais, mais recourt de plus en plus aux produits de l'industrie: colorants alimentaires, gélatine instantanée, etc.

À cet égard, on assiste à l'émergence d'un courant qui tente de (re)dorer le blason de la cuisine faite à partir des produits industriels, auxquels on associe volontiers des qualités gastronomiques. En réponse à une standardisation croissante des produits issus de l'industrialisation du secteur alimentaire et pour pallier la perte de savoir-faire qu'entraîne l'utilisation de ces denrées qui se cuisinent «sans effort», la créativité devient pour la ménagère le lieu où elle peut encore exprimer son talent culinaire, voire sa véritable planche de salut. Selon nous, les critères esthétiques qui se développent dans la cuisine des années 1950 permettent à la fois de maintenir un certain niveau d'exigence culinaire et de continuer à valoriser le travail ménager des femmes. En témoignent ces "paniers de crème glacée» entièrement comestibles, présentés comme un «divertissement gastronomique qui vous attirera des compliments lors de la prochaine réception que vous offrirez en l'honneur du mariage de votre plus chère amie $^{69}$ ».

On le constate: esthétique et sens de l'hospitalité sont deux motivations d'importance dans le travail de la cuisinière "moderne». Cela n'empêche toutefois pas les manuels de standardiser toujours plus les prescriptions liées à la préparation et au service de la nourriture, et par le fait même de contribuer à uniformiser les pratiques. Paradoxe? L'intégration de ces deux tendances en apparence opposées devient plus intelligible si on

68. Comme le reconnaissent plusieurs auteurs, cette décennie renoue avec une "cuisine décorative », datant du XIX ${ }^{e}$ siècle (et inspirée à l'époque par la cuisine française). Erika Endrijonas, op. cit., 160.

69. "Crème glacée en panier», La Gazette des campagnes, 25 juin 1953, 4. 
conçoit la cuisine contemporaine à la fois comme science et art : rigoureusement définie bien qu'ouverte à l'innovation. Le modèle de la cuisinière moderne est donc forgé par cette dualité science/art, constamment mise de l'avant dans les prescriptions culinaires.

Mais comme la gastronomie n'est pas qu'affaire d'apparence, la recherche du raffinement s'exprime aussi par un intérêt accru pour le mariage des saveurs et l'alliance de certains aliments. Cet intérêt se manifeste lui aussi tardivement dans la cuisine québécoise : il n'est pas vraiment perceptible dans les discours avant 1950. Ce raffinement, perceptible surtout dans les journaux, s'exprime par une invitation à "gastronomiser» les mets de l'ordinaire et les produits de l'industrie accessibles au plus grand nombre et non pas uniquement à une élite. Prenons comme exemple cette recette de "poulet à l'italienne» faite de spaghettis à la sauce tomate en boîte et de poulet désossé. Il suffit, explique une chronique culinaire en 1960, de mélanger les deux ingrédients pour obtenir un mets en casserole "exquis et ravissant» qui consacrera "votre réputation de parfaite hôtesse ${ }^{70}$ ». La gastronomie passe donc moins, à cette époque, par la qualité et la finesse des ingrédients sélectionnés, que par l'effort de la ménagère de sortir des sentiers battus. En ce sens, la nouveauté est gage de qualité. Et comme les plus grandes qualités hygiéniques sont attribuées aux aliments industriels, ces derniers représentent une garantie supplémentaire. À l'occasion, on présente aux cuisinières des produits comme le pâté de foie, les fruits de mer, les fromages fins mais ces mets sont exclusivement suggérés pour des occasions de réception et leur présence demeure vraisemblablement exceptionnelle.

Qui dit nouveauté dit aussi «mode». L'art culinaire du $\mathrm{xx}^{\mathrm{e}}$ siècle, particulièrement à partir de 1940-1950, se caractérise par un intérêt manifeste pour les nouvelles tendances en matière de préparation et de consommation alimentaires. À cet égard, les États-Unis occupent une place de choix dans les discours sur la cuisine "moderne». L'American Way of Life qui courtise le Québec dans la première moitié du $\mathrm{xx}^{\mathrm{e}}$ siècle ${ }^{71}$ prend d'assaut la cuisine en proposant des mets rapides, faciles et originaux, mais pas forcément sains.

70. Le Peuple, 15 janvier 1960, 13.

71. Déjà en 1935, l’anthropologue Horace Miner constate, en étudiant les modes de vie du village de Saint-Denis de Kamouraska, que les nouveautés alimentaires sont essentiellement issues de la vie urbaine et américaine. Horace Miner, Saint-Denis : un village québécois (Montréal, Hurtubise HMH, 1985), 302, 369. 
En témoigne la popularité croissante des sodas, des milk shake et autres nourritures de snack bar: "Siroter une limonade était peut-être un rafraîchissement idéal vers 1920, mais en 1954, la mode est changée. Aujourd'hui, on sirote des sodas ${ }^{72}$.» De tels discours nous poussent à analyser l'augmentation de la consommation du sucre à la lumière de cette américanisation. Bien que le Québec, patrie de l'érable à sucre, soit reconnu pour sa "dent sucrée", l'American Sweet Tooth constitue un phénomène de société qui s'étend à tout le continent américain ${ }^{73}$. D'autres exemples montrent le poids des nourritures sucrées dans les nouvelles tendances de l'art culinaire, comme la réception-dessert:

Cette nouvelle mode trouve des adeptes parmi les maîtresses de maison pressées. C'est si facile de préparer un dessert et un breuvage comparé à la tâche de fabriquer quantité de sandwichs, gâteaux et biscuits. Pour cette réception, la jeune hôtesse avertie voudra quelque chose d'élégant mais pas trop difficile à préparer: la crème glacée est la solution. Cette année, la mode est au rose fraise, au beige amande, au rouge cerise et au blanc vanillé ${ }^{74}$.

Ici encore, le domaine de l'art rejoint l'alimentation: visiblement, le mariage des couleurs revêt autant d'importance dans le plateau de crème glacée que dans le domaine vestimentaire ou la décoration intérieure. La même tendance est perceptible dans les manuels d'institution comme $\mathrm{La}$ cuisine raisonnée de 1954: «La note dominante de la mode, en ce $\mathrm{xx}^{\mathrm{e}}$ siècle, est la simplicité de la ligne, tant en peinture, couture ou gastronomie $^{75}$.»

La cuisine est donc le lieu idéal pour une diffusion des dernières tendances, qui puisent beaucoup du côté des États-Unis, il est vrai, mais également à d'autres sources au milieu du $\mathrm{xx}^{\mathrm{e}}$ siècle, notamment aux cultures étrangères ${ }^{76}$.

72. «La mode en 1954», La Gazette des Campagnes, 26 mai 1954, 5.

73. Harvey Levenstein, op. cit., 6. Sidney Mintz parle pour sa part de l'English Sweet Tooth, dont il analyse les fondements. Sidney Mintz, op. cit.

74. «La réception-dessert, une nouvelle mode pratique», loc. cit., 3 juin 1954, 5.

75. Congrégation Notre-Dame, La cuisine raisonnée (1954), chapitre 6: L'art de recevoir à table.

76. Les influences culturelles et la cuisine ethnique ont été traitées au chapitre 2 de notre mémoire. Les courants d'immigration au tournant du siècle et entre 1945 et 1960 ainsi que l’ouverture sur le monde occasionnée par les conflits mondiaux ont été l'occasion de prendre contact avec de nouveaux aliments et de nouvelles cuisines, dont celles de l'Italie et de la Chine dans un premier temps. Cet aspect cadrait moins avec le propos de cet article qui était surtout de montrer comment le discours sur l'art culinaire puise aussi au phénomène de mode et d'urbanisation de la culture. 


\section{CONCLUSION}

La consommation alimentaire est un vaste terrain d'enquête. Elle a été abordée ici sous l'angle des prescriptions adressées aux ménagères dans la littérature culinaire : l'analyse des recettes et des discours sur la cuisine domestique a permis de rendre compte de l'évolution des pratiques de consommation ainsi que de la multiplicité des facteurs qui modulent cette évolution. Le cas du sucre à lui seul nous a permis d'illustrer comment des facteurs d'ordre diététique, social et culturel peuvent expliquer un même phénomène.

Disons-le, la cuisine domestique québécoise a subi entre 1860 et 1960 quelques "révolutions», qui témoignent de transformations majeures au sein de la société : primauté de la science, rationalisation du travail, éclatement de la consommation, accélération du rythme de vie.

Cet article a permis de mettre en lumière l'impact des facteurs scientifiques sur la transformation des habitudes alimentaires. Il a également montré l'existence d'un décalage entre les prescriptions diététiques et les usages, lesquels peuvent traduire une tendance à la baisse de la qualité des nourritures consommées (augmentation du sucre et des graisses dans les recettes), en même temps qu'une sensibilité accrue à l'égard de la santé du corps (valorisation des fruits et légumes crus, recherche de l'équilibre nutritionnel, importance de l'hygiène alimentaire). L'abondance et la diversité accrue des aliments sont deux autres tendances soulevées dans cet article. En témoignent l'augmentation du nombre de variétés de fruits et légumes et l'usage généralisé des nombreux aliments industriels, déclinés sous de multiples variantes et saveurs. Il serait à propos d'approfondir cette question et de la mettre en relation avec des données sur l'ouverture des marchés, les avancées technologiques et agricoles et l'immigration.

Parallèlement à cette offre accrue de produits, une certaine uniformisation des denrées et des usages se fait pourtant jour: étapes de préparation des recettes et techniques enseignées de plus en plus formalisées, standardisation industrielle qui conduit à une uniformisation des produits et, sans aucun doute, des goûts. Par ailleurs, et peut-être en réponse à cette vague uniformisante, on assiste à une élévation des exigences culinaires, notamment sur les plans de l'esthétique et de l'hospitalité. La standardisation des produits impose sans doute à la ménagère de se démarquer à un autre niveau. Encore une fois, il conviendrait de comparer ces nouvelles exigences dans le domaine culinaire avec d'autres domaines de la vie domestique et sociale à la même époque: entretien ménager, éducation familiale, mode vestimentaire et rapport au corps, etc. 
En somme, l'analyse de la littérature culinaire québécoise entre 1860 et 1960 laisse entrevoir une certaine complexification des prescriptions adressées à la ménagère. Une dualité s'exprime dans les requêtes faites aux femmes, qui doivent se montrer à la fois rigoureuses et inventives, économes et généreuses, qui doivent connaître autant les rudiments de la chimie alimentaire que les techniques de glaçage à la mode. Les exigences de la science et de l'art se combinent pour donner lieu à des modèles socioalimentaires renouvelés et plus complexes. À cet égard, "science culinaire» et «art culinaire» se réclament souvent des mêmes postulats, qui tendent vers un idéal ménager où règnent l'ordre, la propreté, la créativité, mais qui est aussi de plus en plus axé vers l'art de vivre et le confort. En somme, les nouvelles conceptions de la cuisine domestique profitent des normes édictées par les sciences ménagères et par les modes alimentaires pour forger un modèle de consommation mettant de l'avant santé et gastronomie. On peut se demander pourquoi plaisir gastronomique et restriction diététique progressent en même temps dans les discours ${ }^{77}$.

À l'aube de la Révolution tranquille, les habitudes culinaires seront grandement modifiées et préfigureront les dernières décennies du $\mathrm{xx}^{\mathrm{e}}$ siècle. Une nouvelle ouverture sur le monde ${ }^{78}$, l'augmentation des voyages à l'étranger, les bouleversements dans les modes de vie ${ }^{79}$, l'explosion de la consommation caractériseront le début de cette période. Une multitude d'options culinaires seront dès lors offertes aux consommateurs. Cuisines du monde, régimes multiples ${ }^{80}$, chaînes de restauration rapide, repas emballés individuellement n'en sont que quelques exemples. Parallèlement, des inquiétudes surgiront quant aux effets de la modernisation des habitudes de consommation et de l'abondance sur le régime alimentaire et susciteront un retour en force de la cuisine au naturel et du terroir, en réaction aux aliments industriels standardisés et, à bien des égards, dénaturés. Émerge également à partir des années 1970 une nouvelle littérature culinaire "régionale» ou "traditionnelle», qui traduit sans doute la crainte de perdre un patrimoine culinaire qui s'étiole. Bref, la porte semble ouverte à tous les courants et à toutes les tendances. Les consommateurs ont de plus en plus l'embarras du choix devant les avenues culinaires qui s'offrent à eux.

77. Claude Fischler, L'Homnivore, op. cit., 240.

78. Notamment avec Expo'67.

79. Famille restreinte, divorce, baisse de la pratique religieuse, explosion des banlieues.

80. Cette expression renvoie autant aux nouveaux concepts en émergence (cuisine santé, cuisine minceur) qu'aux groupes spécialisés dédiés à l'alimentation qui surgissent dans le paysage de la consommation (les Weight Watchers voient le jour en 1961). 